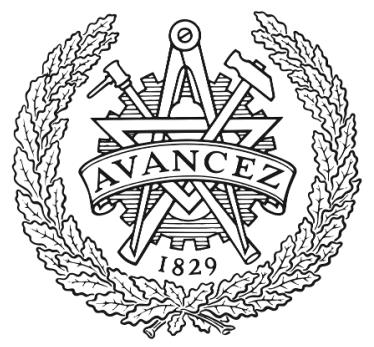

CHALMERS

UNIVERSITY OF TECHNOLOGY

\title{
50-Gb/s Dispersion-unmanaged DMT Transmission with Injection Locked 10G-class 1.55-mu m DML
}

Downloaded from: https://research.chalmers.se, 2023-04-26 09:02 UTC

Citation for the original published paper (version of record):

Xue, L., Yi, L., Zhang, L. et al (2019). 50-Gb/s Dispersion-unmanaged DMT Transmission with Injection Locked 10G-class 1.55-mu m DML. Optics InfoBase Conference Papers. http://dx.doi.org/10.1364/CLEO_SI.2019.SW4O.2

N.B. When citing this work, cite the original published paper. 


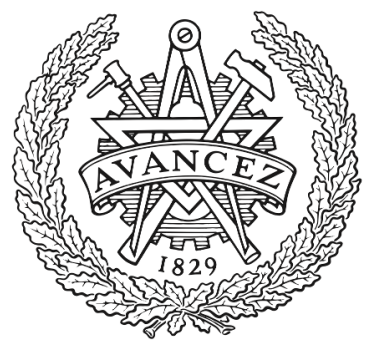

CHALMERS

UNIVERSITY OF TECHNOLOGY

\section{0-Gb/s Dispersion-unmanaged DMT Transmission with Injection Locked 10G-class 1.55- $\mu \mathrm{m}$ DML}

Downloaded from: https://research.chalmers.se, 2019-09-07 22:09 UTC

Citation for the original published paper (version of record):

Xue, L., Yi, L., Zhang, L. et al (2019)

50-Gb/s Dispersion-unmanaged DMT Transmission with Injection Locked 10G-class 1.55- $\mu \mathrm{m}$ DML [Source Title missing]

http://dx.doi.org/10.1364/CLEO_SI.2019.SW4O.2

N.B. When citing this work, cite the original published paper. 


\title{
50-Gb/s Dispersion-unmanaged DMT Transmission with Injection Locked 10G-class 1.55- $\mu \mathrm{m}$ DML
}

\author{
Lei Xue ${ }^{1,3}$, Lilin $\mathrm{Yi}^{3^{*}}$, Lu Zhang ${ }^{2,3}$, Oskars Ozolins ${ }^{2,4}$, Aleksejs Udalcovs ${ }^{4}$, Xiaodan Pang ${ }^{4,5}$, Jiajia Chen $^{2 *}$ \\ ${ }^{I}$ Chalmers University of Technology, Gothenburg, Sweden \\ ${ }^{2}$ KTH Royal Institute of Technology, Stockholm, Sweden \\ ${ }^{3}$ State Key Laboratory of Advanced Optical Communication System and Networks, Shanghai Jiao Tong University, China \\ ${ }^{4}$ Networking and Transmission Laboratory, RISE AB, Stockholm, Sweden \\ ${ }^{5}$ Infinera, Stockholm, Sweden \\ jiajiac@kth.se; lilinyi@sjtu.edu.cn
}

\begin{abstract}
We demonstrate $50-\mathrm{Gb} / \mathrm{s}$ DMT signal transmission over $20-\mathrm{km}$ SMF by using a $10 \mathrm{G}-$ class $1.55-\mu \mathrm{m}$ DML without optical dispersion compensation. Injection locking technique is introduced, which doubles system bandwidth and greatly suppresses DML chirp.

OCIS codes: (060.0060) Fiber optics and optical communications; (060.2360) Fiber optics links and subsystems
\end{abstract}

\section{Introduction}

To fulfill the capacity demand of bandwidth-consuming services while keeping low cost and high energy efficiency, dense wavelength-division multiplexing (DWDM) technique in C-band offers a great potential to support terabit transmission and beyond. From a perspective of implementation, one of the most cost-effective way is the direct detection based C-band transmission, where directly-modulated laser (DML) is preferred thanks to its low cost, large output power, and low energy consumption [1], particularly for short-reach optical communications, such as broadband access and datacenter interconnections. However, the main challenges associated with the DML based Cband optical transmission system is the bandwidth limitation caused by fiber chromatic dispersions in C-band and interplay effects between DML's frequency chirp and chromatic dispersion. Dispersion supported equalization (DSE) [2] and optical filtering based on delay interferometer (DI) [3] have been demonstrated to manage the DML chirp, but DSE is favorable in O-band and DI is sensitive to wavelength shifting.

Injection locking is able to effectively improve the dynamic performance of semiconductor lasers, including the laser modulation bandwidth enhancement and DML's modulation frequency chirp suppression [4]. Moreover, the modulated signal after external injection is vestigial-sideband (VSB), which becomes more tolerant to chromatic dispersion than the double-sideband signal.

In this paper, we introduce injection locking into C-band DML based optical transmissions not only to increase the system bandwidth, but also to enhance the system robustness against chromatic dispersion and semiconductor laser chirp. A direct detection system with discrete multitone (DMT) signals generated by an injection-locked 10Gclass $1.55-\mu \mathrm{m}$ DML over 20 -km dispersion-unmanaged SMF is experimentally carried out. The experimental results show $\sim 4 \mathrm{~dB}$ improvement in signal-to-noise ratio (SNR) and double system capacity (i.e., from $25-\mathrm{Gb} / \mathrm{s}$ to $50-\mathrm{Gb} / \mathrm{s}$ ) by adopting injection locking.

\section{Experiment setup and results}

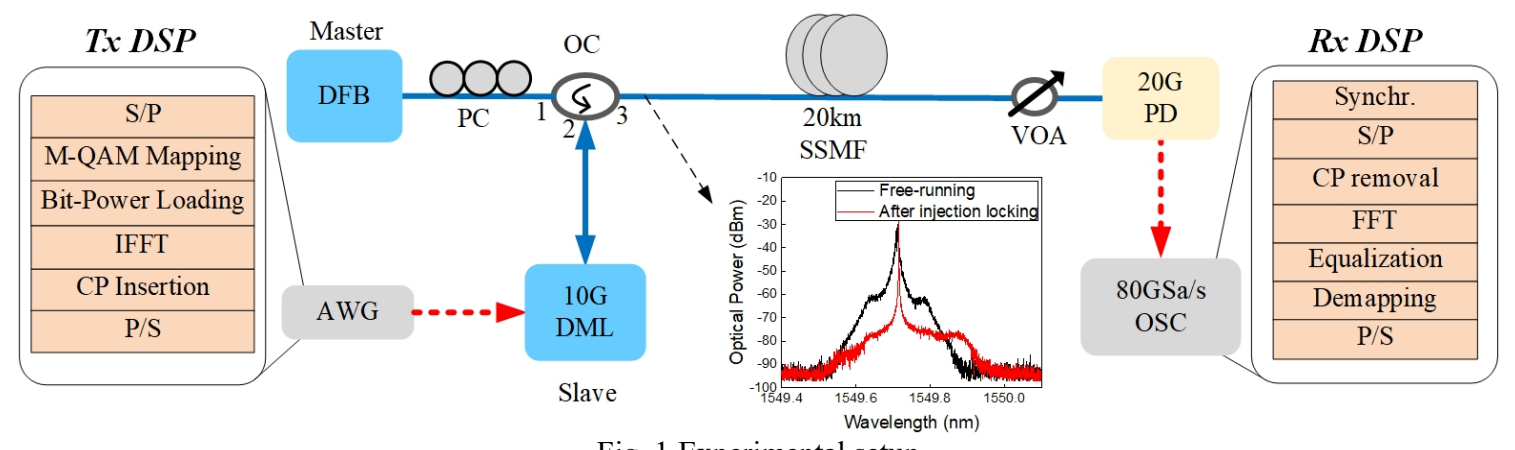

Fig. 1 Experimental setup

Figure 1 shows the experimental setup. The master laser is a C-band wavelength tunable distributed feedback (DFB) laser with a central wavelength of $1549.7 \mathrm{~nm}$, and the slave laser is a commercially-available 10G-class C-band DML with a central wavelength of $1549.6 \mathrm{~nm}$. The optical output of the DFB is injected into the DML via a polarization controller (PC) and optical circulator (OC). The PC is employed to match the polarization between two 
lasers to ensure the stability of injection locking. The optical spectrum of the DML modulated with 25-Gbaud signal before and after injection locking is shown in the inset of Fig. 1. The chirp that induces the broadened spectrum is significantly suppressed after injection locking. Besides, a power gain due to the red-shift cavity mode at the longer wavelength improves the frequency response after PD detection as well as efficiency of VSB modulation. The narrowed spectrum and VSB modulation enhance the system tolerance of fiber chromatic dispersion. Although the modulation depth after injection is degraded, it could be optimized by tuning the wavelength difference between the master and slave lasers and/or the injection power.

In the experiments, the DMT signal is first generated offline and loaded onto the 50-GSa/s arbitrary waveform generator (AWG). Then the $50-\mathrm{Gb} / \mathrm{s}$ DMT signal with an amplitude of $1.5-\mathrm{V}$ is modulated to the injection-locked DML. After 20-km SMF transmission, the optical signal is detected by a $20-\mathrm{GHz}$ p-i-n photodetector (PD) with integrated transimpedance amplifier (TIA). The received optical power is varied by a variable optical attenuator (VOA). The detected electrical signal is sampled by an $80-\mathrm{GSa} / \mathrm{s}$ digital storage scope with analog bandwidth of 33GHz.

We employ the digital signal processing (DSP) flow used in [5]. In the transmitter, the bits and power loading is utilized to enhance the capacity. In the experiments, the length of the inverse fast Fourier transform (IFFT) and cyclic prefix (CP) are set to 1024 and 16, respectively. The equalization, including one-tap linear equalization and Volterra nonlinear equalization, is utilized to compensate the system distortion.
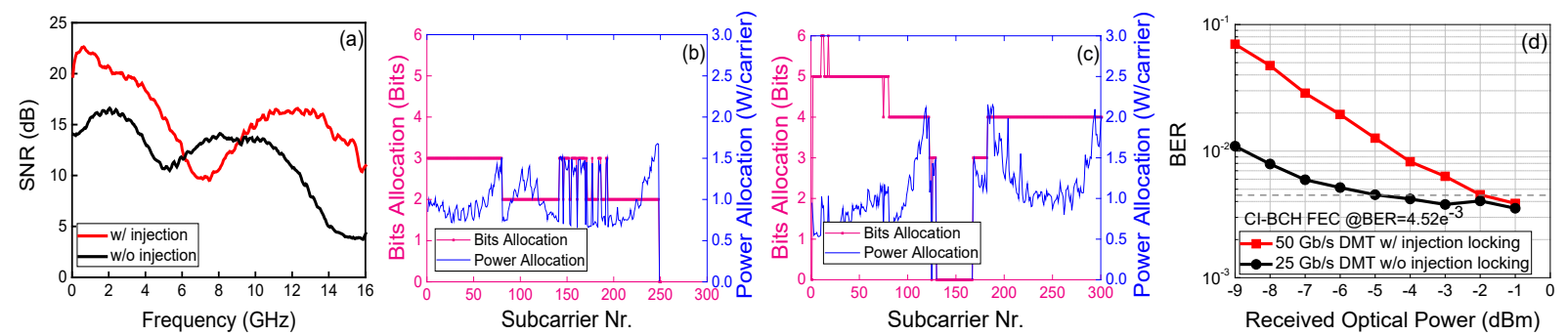

Fig. 2 SNR versus frequency with and without injection locking (a), bits and power loading profiles without (b) and with injection (c), BER versus received optical power (d) after 20-km SMF transmission

To evaluate the system performance, we first measure the SNR after 20-km SMF with and without injection locking using 16-QAM modulated DMT signal carrying 330 modulated subcarriers, as shown in Fig. 2(a). The average SNR is improved from $11.62 \mathrm{~dB}$ to $15.61 \mathrm{~dB}(\sim 4 \mathrm{~dB}$ increase) thanks to injection locking. The SNR notch due to the fiber dispersion can be improved from $5 \mathrm{GHz}$ to $7 \mathrm{GHz}$ after injection locking, which means the system becomes more tolerant to fiber dispersion. According to the SNR performance at different subcarrier, adaptive bits and power loading is used to further improve the system capacity. Figure 2(b) and (c) depict the bits and power loading profiles of 20-km SMF transmission based on the probed SNR as shown in Fig. 2(a). It can be observed that more bits are allocated at low frequency region and more power are allocated in high frequency region to compensate the channel loss. The transmission performance with and without injection locking after 20-km SMF transmission is shown in Fig. 2(d). Without injection locking, only 25-Gb/s DMT transmission of free-running DML can be achieved at continuously-interleaved Bose-Chaudhuri-Hocquenghem FEC (CI-BCH $(1020,988)$ ) [6] limit (with a bit error rate BER of 4.52E-3). After external injection, 50-Gb/s DMT signal transmission with a BER lower than the CI-BCH FEC limit is achieved, demonstrating that the system capacity can be doubled when optical injection locking is adopted.

\section{Conclusion}

We have experimentally demonstrated the merits of adopting injection locking technique in compensating the bandwidth limitation and distortion induced by DML chirp and fiber dispersion. A 50-Gb/s DMT transmission over $20-\mathrm{km}$ SMF by using a $10 \mathrm{G}$-class $1.55-\mu \mathrm{m}$ DML have been achieved without using any dispersion compensations. Acknowledgements: This work is funded by the EU H2020 5G STEP-FWD (grant 722429), the SJTU-KTH seed grant and VR "PHASE" grant.

\section{References}

[1] J. Yu et al., " $56 \mathrm{~Gb} / \mathrm{s}$ Chirp-managed Symbol Transmission with Low-cost, 10-G Class LD for 400G Intra-data Center Interconnection," OFC2017, paper W4D.2.

[2] L. Xue et al., "50-Gb/s TDM-PON Based on 10G-Class Devices by Optics-simplified DSP," OFC2018, paper M2B.4.

[3] L.Yi et al., "Field-trial of a real-time $100 \mathrm{~Gb} / \mathrm{s}$ TWDM-PON based on 10G-class optical device,"ECOC2016, paper W3.E.1

[4] L. Chrostowski et al, "Microwave performance of optically

injection-locked VCSELs," IEEE Trans. Microw. Theory Tech.54(2), pp.788 (2006).

[5] L. Zhang et al., "Nonlinearity Tolerant High-speed DMT Transmission with 1.5- $\mu \mathrm{m}$ Single-mode VCSEL and Multi-core Fibers for Optical Interconnects," J. Lightwave Technol. (2018).

[6] M. Scholten et al., "Continuously- interleaved BCH (CIBCH) FEC delivers best in class NECG for $40 \mathrm{G}$ and $100 \mathrm{G}$ metro applications," OFC2010, paper NTuB3. 